\title{
Stress evolution during growth of 1-d island arrays: kinetics and length scaling
}

\author{
E. Chason ${ }^{1}$, A.M. Engwall ${ }^{1}$, C.M. Miller ${ }^{1}$, C. - H. Chen $^{1}$, A. Bhandari ${ }^{2}$, S.K. Soni ${ }^{3}$, S.J. \\ Hearne $^{4}$, L.B. Freund ${ }^{5}$, B.W. Sheldon ${ }^{1}$ \\ ${ }^{1}$ Brown University, School of Engineering, Providence, RI 02912 \\ ${ }^{2}$ PPG Industries, One PPG Place Pittsburgh, PA 15272 \\ ${ }^{3}$ Intel Corporation, 5200 NE Elam Young Parkway, Hillsboro, OR, 97124 \\ ${ }^{4}$ Sandia National Laboratory, Albuquerque, NM 87185 \\ ${ }^{5}$ University of Illinois a t Urbana-Champaign, Department of Mat erials S cience and \\ Engineering, Urbana, IL 61801
}

\begin{abstract}
To explore the mechanisms controlling residual stress in thin films, we have measured the stress evolution during electrodeposition of $\mathrm{Ni}$ on lithographically patterned substrates with different pattern spacings and growth rates. Studying films with a controlled island geometry allows us to relate the stress (measured using wafer curvature) to the evolution of the morphology. We analyze the measurements with a model that focuses on the stress that develops where adjacent islands grow together to form new elements of grain boundary.
\end{abstract}

Residual stress in polycrystalline thin films is a persistent problem, since it can significantly reduce film performance or lead to failure [1]. A deeper understanding would enable it to be predicted and controlled.

Numerous studies have shown how the stress evolution depends on the material, processing conditions and evolving microstructure (many studies are reviewed in $[2,3,4]$ ). Films with low atomic mobility tend to grow in a state of tensile stress while films with higher mobility are compressive. Similarly, raising the growth temperature [5] or decreasing the growth rate [6] can change the stress from tensile to compressive. During growth, the stress goes through multiple states that correspond with the evolving microstructure, i.e., from isolated nuclei (low or compressive stress) through island coalescence (tensile stress) to a continuous film (steady-state stress that depends on growth rate) [7].

Various mechanisms have been proposed to explain different aspects of the stress evolution. Compressive stress in the nucleation stage has been attributed to lattice compression induced by the surface stress [8]. Hoffman [9] proposed that the tensile stress arises due to attractive forces between islands when the grain boundary forms, similar to the reason for cracks closing [10]. The origin of the post-coalescence compressive stress is more controversial, with various groups attributing it to: adatoms on 
the surface [11], stress from the pre-coalescence stage [12], trapping of atoms between surface steps [13] and the insertion of atoms into the grain boundary during growth [14].

We have recently proposed a model [15] (described below) that focuses on the stress that develops at the point where layers in adjacent grains grow together to form new segments of grain boundary. Each layer's stress is predicted to depend on the rate at which the grain boundary height is changing when it forms. However, validation of this model is difficult because the shape of individual islands (and hence the rate of grain boundary formation) is not known during the film growth. To overcome this problem, we have grown patterned arrays of islands in which the geometry during growth is known $[16,17,18]$. In the current work, we study stress evolution in a linear array in which the islands grow in the form of half-cylinders. Films with different pattern spacings $(L)$ and growth rates $(R)$ are measured to compare with the analytical model.

The samples consisted of Ni thin films electrodeposited on 100 micron thick Si substrates with $15 \mathrm{~nm}$ Ti and $150 \mathrm{~nm}$ Au layers deposited by electron beam evaporation on native oxide. After coating with photoresist, the substrate was lithographically patterned with linear trenches that exposed the Au underlayer for subsequent electrodeposition. Deposition was performed from a nickel sulphamate/boric acid bath; further details of the patterning and deposition process can be found in ref. [17].

Substrates were prepared with trench arrays spaced apart 5.3, 10.6, or $26.5 \mu \mathrm{m}$, with corresponding trench widths of 2,4 , and $10 \mu \mathrm{m}$. Films were grown under potentiostaic control at multiple growth rates for each pattern spacing. Under these conditions, the growth rate normal to the surface is nearly constant so that each line grows into the form of a half-cylinder before it impinges on the one next to it. After the lines grow large enough to intersect (shown schematically in figure 1), they continue to grow with a constant radial growth rate. The inset shows a scanning electron microscope (SEM) image of the morphology. The focused ion beam (FIB) cross-section shows that the shape of each island is semicircular and consists of multiple grains.

The evolution of the stress was measured using a real-time multi-beam wafer curvature technique (MOS) [4] during deposition. To relate the measured curvature to the film stress, we must account for the fact that the patterned films are not spatially uniform or rotationally symmetric in the plane of the film. As shown in figure 1, the film is assumed to consist of an array of islands that are semi-circular in cross-section with radius $r$ spaced by $L$ in the $x$-direction. In the y-direction, the film is translationally symmetric. For $r>L / 2$, the islands overlap so that a planar boundary forms between them, analogous to the grain boundary that forms in a polycrystalline film. We define the height of the boundary as $h_{g b}$. The curvature is measured along the $x$-direction $\left(\kappa_{x}\right)$, i.e., normal to the interface that forms between adjacent lines.

We relate the measured curvature to the stress following the approach described in Freund and Suresh [19] for anisotropic films:

$$
\kappa_{x}=\frac{6\left(f_{x}-v_{s} f_{y}\right)}{E_{s} h_{s}^{2}}
$$

where $E_{s}, \boldsymbol{v}_{s}$ and $h_{s}$ are the elastic modulus, Poisson's ratio and thickness of the substrate, respectively. $f_{x}$ and $f_{y}$ are due to the stress components in the film in the $x$ - and $y$ direction respectively. It is the deformation they induce plus compatibility that results in 
substrate curvature. For a uniform film, $f_{x}=f_{y}=\langle\boldsymbol{\sigma}\rangle h_{f}$ where $\langle\boldsymbol{\sigma}\rangle$ is the average equibiaxial stress and $h_{f}$ is the film thickness. In this case, the typical Stoney's formula is recovered.

The force in the $x$-direction $\left(f_{x}\right)$ can be found by integrating the stress normal to the boundary between the lines $\left(\sigma_{x x}\right.$ at $\left.x=L / 2\right)$ over the thickness of the film. This stress is independent of the $y$-position so that $f_{x}$ is given by:

$$
f_{x}=<\sigma>_{x x} h_{f}=\int_{0}^{h_{g b}} \sigma_{x x}\left(x=\frac{L}{2}, z\right) d z
$$

Here we define $<\sigma_{x x}>$ as the average normal stress in the $x$-direction.

Similarly, we can compute $f_{y}$ due to stress in the $y$-direction along the length of the lines. Integrating over the cross-sectional area of the film gives:

$$
f_{y}=\langle\sigma\rangle_{y y} h_{f}=\frac{1}{L} \int_{0}^{\infty} \int_{-L / 2}^{L / 2} \sigma_{y y}(x, z) d x d z
$$

where we define $<\sigma_{\mathrm{yy}}>$ as the average normal stress in the y-direction.

Eq. 1 is valid when the thickness of the film is much less than the thickness of the substrate. When this is not the case, Freund et al. [20] have calculated a correction factor relating the measured curvature to the curvature expected from the Stoney equation. Although this formula was originally derived for a film that is spatially uniform and isotropic, we use it for the patterned films following ref [17]:

$$
\kappa=\kappa_{S T}\left(1+\frac{h_{f}}{h_{s}}\right)\left[1+4 \frac{h_{f}}{h_{s}} \frac{\bar{E}_{f}}{\bar{E}_{s}}+6 \frac{h_{f}^{2}}{h_{s}^{2}} \frac{\bar{E}_{f}}{\bar{E}_{s}}+4 \frac{h_{f}^{3}}{h_{s}^{3}} \frac{\bar{E}_{f}}{\bar{E}_{s}}+\frac{h_{f}^{4}}{h_{s}^{4}} \frac{\bar{E}_{f}^{2}}{\bar{E}_{s}^{2}}\right]^{-1}
$$

$\overline{E_{f}} / \overline{E_{S}}$ is the ratio of the plane strain moduli for the film and substrate, which is taken here as 1.4 [17]. The measured curvature $(\kappa)$ was divided by this correction factor to obtain the curvature that would be expected from a thin anisotropic film.

Measurements of the curvature vs. time are shown in figure 2 for pattern spacings of a) 5.3 , b) 10.6 and c) 26.5 microns. The growth rates for each measurement are indicated on the figure. The curvature has been multiplied by $E_{s} h_{s}{ }^{2} / 6$ so that it is equal to $\left(<\sigma_{x x}>-v_{s}<\sigma_{y y}>\right) h_{f}$, referred to as the stress-thickness.

The evolution of the stress-thickness has similar features for all of the measured conditions. When the radius is less than $L / 2$, parallel islands do not make contact and the change in the stress-thickness is relatively small. After they start to impinge, the stress- 
thickness rises rapidly. Subsequently, the slope of the stress-thickness decreases until it reaches a steady-state with a relatively constant slope. For the faster growth rates, the steady-state slope is positive (corresponding to tensile stress) while for slower growth rates the slope becomes less tensile or even compressive. This behavior is similar to that seen in polycrystalline unpatterned films, but the coalescence here occurs at much larger film thickness because the patterned island spacing is much larger than the typical grain size in unpatterned polycrystalline films.

In order to interpret these results, we use a model developed for stress in polycrystalline films [15]. It has been described previously so it will only be discussed briefly here. The model is based on rate equations that describe the stress-generating processes that occur in each layer (indexed by the letter $i$ ) at the evolving grain boundary. We assume that the stress in individual layers in the film is independent of the stress in other layers (which Guduru refers to as a linear spring model [21]). The model contains two competing mechanisms of tensile and compressive stress generation. The tensile stress $\left(\sigma_{T}\right)$ is based on Hoffman's model [9] and develops due to the attraction between adjacent layers as they grow towards each other. It depends on the difference between the surface and interfacial energies and the spacing between the grain boundaries (as $\left.1 / L^{1 / 2}\right)$; other approaches based on crack closure [10] and contact mechanics [22] give similar results. The compressive stress is assumed to be due to the insertion of atoms into the grain boundary $\left(N_{i}\right)$ driven by the difference between the chemical potential for atoms on the surface and in the grain boundary. The resulting normal stress in each layer is due to the sum of the tensile and compressive stresses:

$$
\sigma_{x x, i}=\sigma_{T}-M_{f} \frac{N_{i} a}{L}
$$

where $M_{f}$ is the biaxial modulus of the film and $a$ is the nominal height of an atomic layer.

The rate at which atoms are inserted into the grain boundary $\left(d N_{i} / d t\right)$ is due to the chemical potential difference $(\Delta \mu)$ between atoms on the surface $\left(\mu_{s}\right)$ and atoms in the grain boundary $\left(\mu_{g b}\right)$ during growth. The chemical potential of atoms on the surface is assumed to be elevated by an amount $\delta \mu_{s}$ above the equilibrium value, e.g., due to supersaturation induced by the deposition flux. In addition, stress changes the chemical potential for atoms in the grain boundary so that the net chemical potential difference is:

$$
\Delta \mu \equiv \mu_{S}-\mu_{g b}=\delta \mu_{S}+\frac{1}{3}\left(\sigma_{x x, i}+\sigma_{y y, i}\right) \Omega
$$

where $\Omega$ is the nominal atomic volume. This is assumed to drive a flux of atoms into the grain boundary as the film grows.

Note that we have modified eq. 6 from previous treatments to allow stress anisotropy in the film. Because the thickness is much larger than the polycrystalline grain size, the film stress in the $y$-direction is expected to reach its steady-state value. We 
therefore assume that it has a constant value $\left(\sigma_{y y, s s}\right)$ that does not depend on the layer index $i$.

Putting these mechanisms together results in a differential equation for the evolution of stress in the $i^{\text {th }}$ layer:

$$
\frac{\partial \sigma_{x x, i}}{\partial t}=\frac{-\beta D}{a L}\left(\sigma_{x x, i}-\sigma_{c}\right)
$$

where

$$
\beta \equiv \frac{4 C_{S} M_{f} \Omega}{3 k T}
$$

and

$$
\sigma_{C} \equiv \sigma_{c, o}-\sigma_{y y, s s}
$$

$\sigma_{c, 0}$ is the compressive stress due to the supersaturation $\left(-3 \delta \mu_{s} / \Omega\right), C_{s}$ is the surface concentration of mobile species, $T$ is temperature, and $D$ is the effective diffusivity for transitions from the surface into the grain boundary.

This has a solution of the form

$$
\sigma_{x x, i}\left(\Delta t_{i}\right)=\sigma_{C}+\left(\sigma_{T}-\sigma_{C}\right) \cdot \exp \left(-\frac{\beta D}{a L} \Delta t_{i}\right)
$$

where $\Delta t_{i}$ is the time interval during which the grain boundary in this layer is at the surface. This time interval is given by

$$
\Delta t_{i}=\frac{a}{\frac{d h_{g b}(i)}{d t}}
$$


where $\left(d h_{g b}(i) / d t\right)$ is the rate at which the grain boundary is growing. Physically, this means that when the grain boundary grows quickly (small $\Delta t_{i}$ ), there is not time to insert atoms into it and the stress remains tensile. At slower growth rates (larger $\Delta t_{i}$ ), atoms can be inserted into the grain boundary and the stress becomes more compressive.

Eqs. 8 and 9 predict the stress in each layer due to the interaction between adjacent half-cylinders. Using the island geometry and growth rate, we can calculate the grain boundary velocity as a function of time and then integrate the resulting stress to obtain $f_{x}$. The effect of stress in the $y$-direction is included by multiplying $\boldsymbol{\sigma}_{y y, s s}$ by the cross-sectional area of the film in the $x-z$ plane to calculate $f_{y}$. This tensile stress in the $y-$ direction explains the negative slope in the data before island coalescence occurs.

A non-linear least squares routine is used to fit the model to the data and obtain values for the unknown parameters. The results of the fitting are shown as the solid lines in figure 2. For all of the data fitting we use a single value for the compressive stress due to supersaturation $\left(\sigma_{c, 0}=-20.1 \mathrm{MPa}\right)$ and we allow $\beta D$ to vary within a range of $5 \%$ $\left(4920+/-210 \mathrm{~nm}^{2} / \mathrm{s}\right)$. For each of the pattern spacings $(L=5.6,10.3$ and 26.5$)$, we allow there to be a different value of $\sigma_{\mathrm{T}}(85.7,51.9$ and $34.2 \mathrm{MPa}$, respectively). We also allow there to be a small time offset for each data set, to make the increase in stress at coalescence coincide with the measured rise in the stress. The values of $\boldsymbol{\sigma}_{y y, s s}$ obtained from the fitting for each growth rate are similar to measurements of unpatterned Ni films; their contribution to the measured curvature is small.

The model captures the evolution of the stress for the different growth rates as well as for different pattern spacings. We present the results of using only a very limited set of parameters in order to show how the model can handle a wide range of processing conditions (in $R$ and $L$ ) with a single parameter set. The biggest discrepancy occurs at the lowest growth rates with the largest pattern spacing where the model predicts more tensile stress than seen experimentally. This may be partially due to inaccuracy in the growth rate estimates we are using at low rates. Better fits for the individual data sets can be obtained by letting some of the parameters float independently for each data set. The calculated stress-thickness also rises faster than the measured values for some growth rates. This may be due to imperfections in the lithography so that adjacent islands coalesce at different times, broadening the data. Similarly, the actual island geometry may not conform exactly to the semicircular cross-section we use in the calculations.

The fitting procedure produces values of $\sigma_{\mathrm{T}}$ for each pattern spacing as shown in figure 3 a. For comparison, a fit to $1 / L^{1 / 2}$ (the prediction of the Hoffman model) is shown as the solid line. The experimental results are consistent with this scaling, however the range of the measurements is small and other power law values would also provide reasonable agreement.

For large film thickness, $d h_{g b} / d t$ approaches $R$ so that $\Delta t_{i} \approx a / R$ and the stress is predicted to reach a steady-state. The steady-state stresses estimated from the stressthickness data are shown in figure $3 \mathrm{~b}$. A least-squares fit to the model in the steady-state regime is shown as the solid lines in the figure (with component $\sigma_{y y, s s}$ as a function of $R$ also estimated from the model). The values of the parameters are: $\sigma_{\mathrm{C}, 0}=-5.0 \mathrm{MPa}, \beta D$ $=9648 \mathrm{~nm}^{2} / \mathrm{s}$ and $\sigma_{\mathrm{T}}=161,113$ and $72 \mathrm{MPa}$ for increasing values of $L$. These fitting parameters are slightly different than the ones described above because they were optimized to fit only the steady-state stress and not the entire measurement. They are therefore less sensitive to the early-stage features of the stress evolution. 
In summary, we have measured patterned thin films to study the origin of stress in thin films. Growing linear arrays of islands lets us measure the stress that results from the coalescence of islands with a controlled morphology. The data was fit with a model that focuses on the stress that develops at the triple junction where adjacent islands grow together to form new segments of grain boundary. The model was able to fit the data for multiple spacings and growth rates with a single set of parameters. The tensile stress that occurs due to island coalescence was determined to be consistent with the model proposed by Hoffman.

The authors gratefully acknowledge useful input from Allan Bower, Fei Pei, Brittni Thomas and Julia Zaskorski. This work was supported by the Office of Basic Energy Sciences, Division of Materials Sciences and Engineering under Award \#DESC0008799. Sandia National Laboratories is a multi-program laboratory managed and operated by Sandia Corporation, a wholly owned subsidiary of Lockheed Martin Corporation, for the U.S. Department of Energy's National Nuclear Security Administration under contract DE-AC04-94AL85000.

\section{References}

[1] A. González-González, C. Polop, E. Vasco, Physical Review Letters, 110 (2013) 056101 .

[2] M.F. Doerner, W.D. Nix, Critical Reviews in Solid State and Materials Sciences, 14 (1988) 225-268.

[3] R. Koch, Journal of Physics: Condensed Matter, 6 (1994) 9519.

[4] E. Chason, Thin Solid Films, 526 (2012) 1-14.

[5] R. Abermann, Vacuum, 41 (1990) 1279-1282.

[6] S.J. Hearne, J.A. Floro, Journal of Applied Physics, 97 (2005) 014901.

[7] J.A. Floro, S.J. Hearne, J.A. Hunter, P. Kotula, E. Chason, S.C. Seel, C.V. Thompson, Journal of Applied Physics, 89 (2001) 4886-4897.

[8] R.C. Cammarata, T.M. Trimble, D.J. Srolovitz, Journal of Materials Research, 15 (2000) 2468-2474.

[9] R.W. Hoffman, Thin Solid Films, 34 (1976) 185-190.

[10] W.D. Nix, B.M. Clemens, Journal of Materials Research, 14 (1999) 3467-3473.

[11] C. Friesen, C.V. Thompson, Physical Review Letters, 89 (2002) 126103.

[12] R. Koch, D. Hu, A.K. Das, Physical Review Letters, 94 (2005) 146101. 
[13] F. Spaepen, Acta Materialia, 48 (2000) 31-42.

[14] E. Chason, B.W. Sheldon, L.B. Freund, J.A. Floro, S.J. Hearne, Physical Review Letters, 88 (2002) 156103.

[15] E. Chason, J.W. Shin, S.J. Hearne, L.B. Freund, Journal of Applied Physics, 111 (2012) 083520.

[16] S.J. Hearne, S.C. Seel, J.A. Floro, C.W. Dyck, W. Fan, S.R.J. Brueck, Journal of Applied Physics, 97 (2005) 083530.

[17] A. Bhandari, B.W. Sheldon, S.J. Hearne, Journal of Applied Physics, 101 (2007) 033528 .

[18] E. Chason, J.W. Shin, C.-H. Chen, A.M. Engwall, C.M. Miller, S.J. Hearne, L.B. Freund, Journal of Applied Physics, 115 (2014) 123519.

[19] L.B. Freund, S. Suresh, Thin Film Materials, Cambridge University Press, 2003.

[20] L.B. Freund, J.A. Floro, E. Chason, Applied Physics Letters, 74 (1999) 1987-1989.

[21] P.R. Guduru, E. Chason, L.B. Freund, Journal of the Mechanics and Physics of Solids, 51 (2003) 2127-2148.

[22] L.B. Freund, E. Chason, Journal of Applied Physics, 89 (2001) 4866-4873. 


\section{Figure Captions}

Figure 1. Schematic of island geometry used for analyzing stress evolution. (inset) SEM image of Ni island array with front surface prepared by FIB cross-section.

Figure 2. Stress-thickness vs. time measured on island arrays with spacing of (a) 5.3 (b) 10.6 and (c) $26.5 \mu \mathrm{m}$. The growth rate used for each measurement is indicated on the figure. The solid lines are the result of fits to the model described in the text.

Figure 3. a) Dependence of the tensile stress parameter (derived from the fitting procedure used for the data in figure 2) on the pattern spacing. The solid line is a fit to $\mathrm{L}^{-1 / 2}$. b) Steady-state stress vs. the product of the growth rate and pattern spacing. Pattern spacing for each data set is indicated on figure. The solid lines are fits to the model described in the text. 


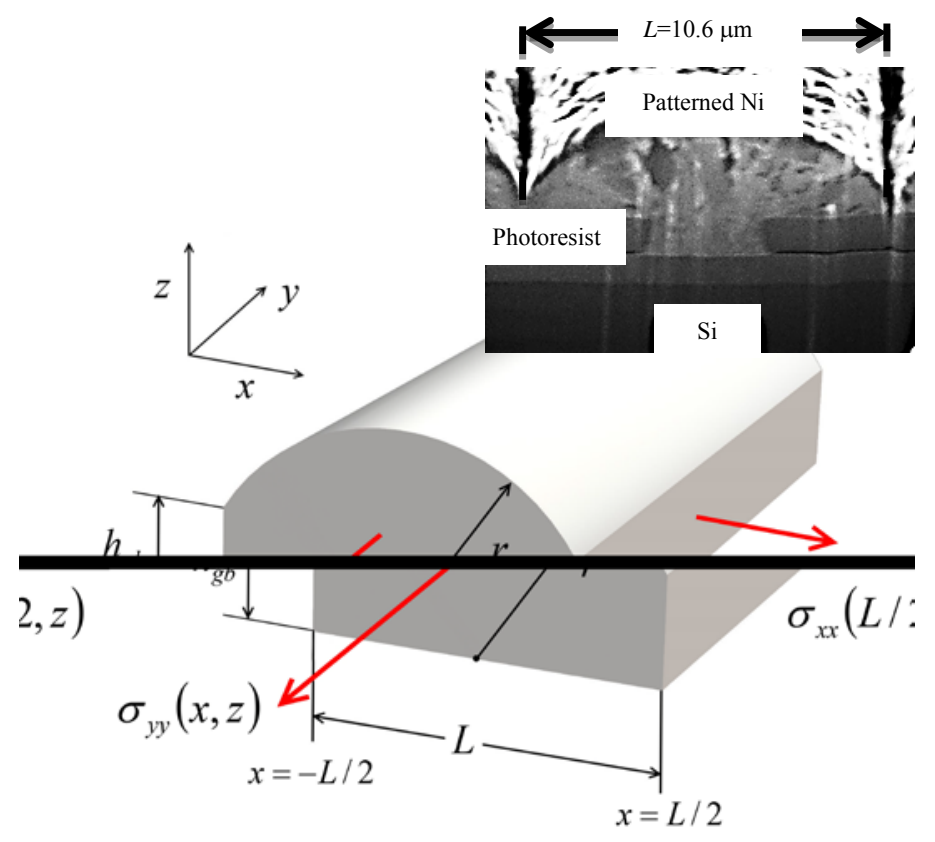

Figure 1. 

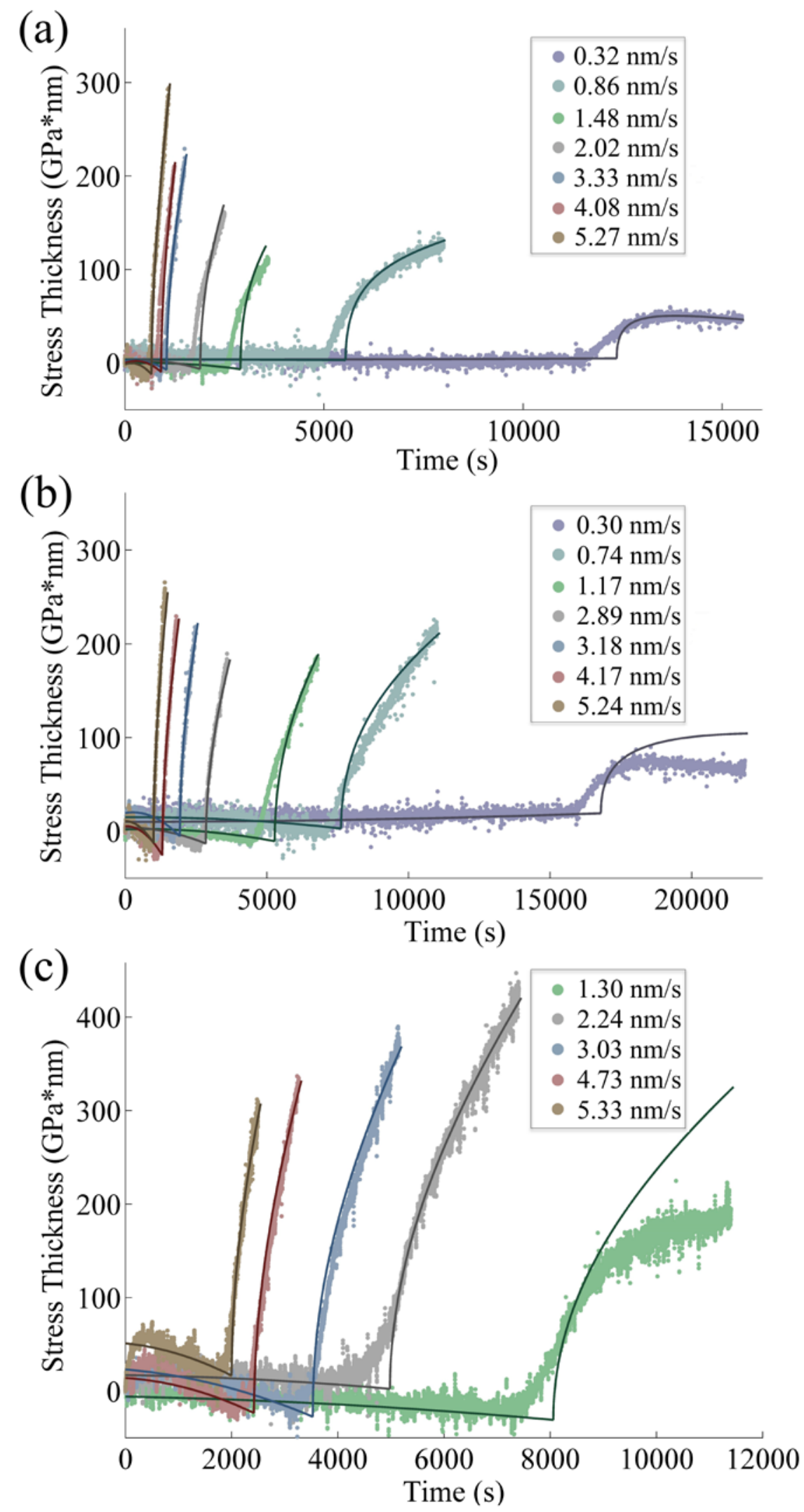

Figure 2. 

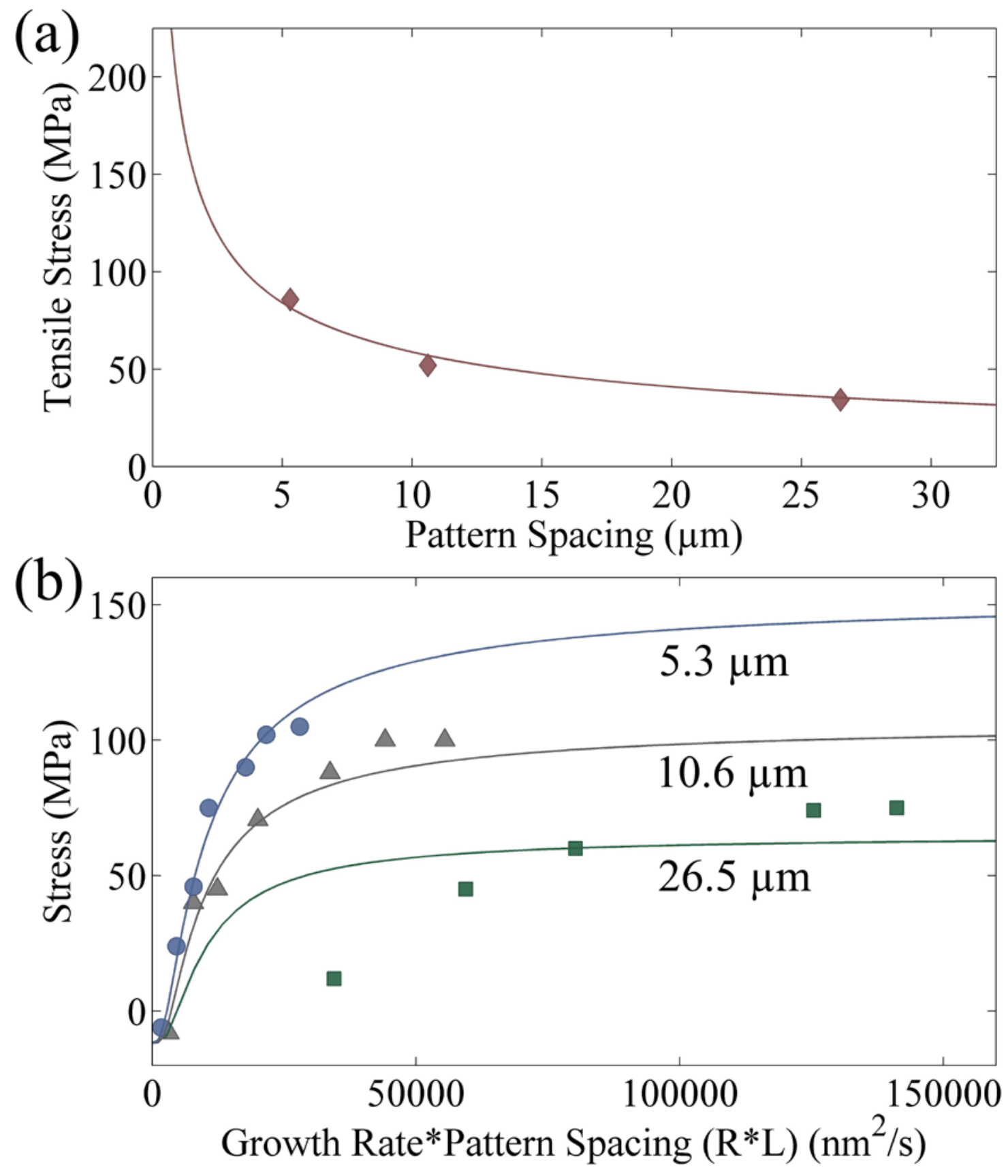

Figure 3. 